Kragujevac Journal of Mathematics

Volume 45(4) (2021), Pages 531-542.

\title{
OSCILLATION CRITERIA FOR SECOND ORDER IMPULSIVE DELAY DYNAMIC EQUATIONS ON TIME SCALE
}

\author{
GOKULA NANDA CHHATRIA ${ }^{1}$
}

\begin{abstract}
In this work, we study the oscillation of a kind of second order impulsive delay dynamic equations on time scale by using impulsive inequality and Riccati transformation technique. Some examples are given to illustrate our main results.
\end{abstract}

\section{INTRODUCTION}

Consider a class of second order impulsive nonlinear dynamic equations of the form:

$(E)\left\{\begin{array}{l}{\left[r(t)\left(x^{\Delta}(t)\right)^{\gamma}\right]^{\Delta}+q(t) x(\sigma(t)-\delta)=0, \quad t \in \mathbb{J}_{\mathbb{T}}:=[0, \infty) \cap \mathbb{T}, t \neq \tau_{k}, t \geq t_{0},} \\ x\left(\tau_{k}^{+}\right)=M_{k}\left(x\left(\tau_{k}\right)\right), \quad x^{\Delta}\left(\tau_{k}^{+}\right)=N_{k}\left(x^{\Delta}\left(\tau_{k}\right)\right), \quad k \in \mathbb{N}, \\ x\left(t_{0}^{+}\right)=x_{0}, \quad x^{\Delta}\left(t_{0}^{+}\right)=x_{0}^{\Delta}, \quad t_{0}-\delta \leq t \leq t_{0},\end{array}\right.$

under the following hypotheses.

$\left(A_{1}\right) \gamma \geq 1$ is the quotient of odd positive integers, $\mathbb{T}$ is an unbouned above time scale with $0 \in \mathbb{T}$ and $\tau_{k} \in \mathbb{T}$ satisfying the properties $0 \leq t_{0}<\tau_{1}<\tau_{2}<\cdots<$ $\tau_{k}, \lim _{k \rightarrow \infty} \tau_{k}=\infty$,

$$
x\left(\tau_{k}^{+}\right)=\lim _{h \rightarrow 0^{+}} x\left(\tau_{k}+h\right), \quad x^{\Delta}\left(\tau_{k}^{+}\right)=\lim _{h \rightarrow 0^{+}} x^{\Delta}\left(\tau_{k}+h\right),
$$

which represent the right limit of $x(t)$ at $t=\tau_{k}$ in the sense of time scale. If $\tau_{k}$ is right scattered, then $x\left(\tau_{k}^{+}\right)=x\left(\tau_{k}\right), x^{\Delta}\left(\tau_{k}^{+}\right)=x^{\Delta}\left(\tau_{k}\right)$. Similarly, we can define $x\left(\tau_{k}^{-}\right), x^{\Delta}\left(\tau_{k}^{-}\right)$.

$\left(A_{2}\right) \delta \in \mathbb{R}_{+}, \sigma(t)-\delta \in \mathbb{T}, r(t)>0, q(t) \in C_{r d}\left(\mathbb{T},\left[t_{0}, \infty\right)_{\mathbb{T}}\right)$.

Key words and phrases. Oscillation, delay dynamic equation, impulse, time scales. 2010 Mathematics Subject Classification. Primary: 34A37. Secondary: 34A60, 39A12.

DOI 10.46793/KgJMat2104.531C

Received: January 29, 2019.

Accepted: Match 12, 2019. 
$\left(A_{3}\right) M_{k}, N_{k}: \mathbb{R} \rightarrow \mathbb{R}$ are continuous functions, $M_{k}(0)=0=N_{k}(0)$ and there exist numbers $a_{k}, a_{k}^{*}, b_{k}, b_{k}^{*}$ such that $a_{k}^{*} \leq \frac{M_{k}(u)}{u} \leq a_{k}, b_{k}^{*} \leq \frac{N_{k}(u)}{u} \leq b_{k}, u \neq 0$, $k \in \mathbb{N}$.

In this work, our objective is to extend the work of [15] to the second order impulsive delay dynamic equations $(E)$. About the time scale concept and fundamentals of time scale calculus we refer the monographs [6] and [7].

Oscillation theory of impulsive differential/difference equation has brought the attention of many researchers, as it provides a more adequate mathematical model for numerous process and phenomena studied in physics, biology, engineering and to mention a few. In the literature, most of the results obtained for difference equations is the continuous analogues of differential equations and vice versa. Hence it was an immediate question to find a way for which one can unify the qualitative properties of both equations. In 1988 Stefen Hilger introduced the concept of time scales calculus, which unify the continuous and discrete calculus in his Ph.D. thesis [12]. The study of impulsive dynamic equations on time scales has been initiated by Benchora et al. [4]. form

In [15], Huang has considered the second order impulsive dynamic equation of the

$$
\left\{\begin{array}{l}
{\left[r(t)\left(y^{\Delta}(t)\right)^{\gamma}\right]^{\Delta}+f\left(t, y^{\sigma}(t)\right)=0, \quad t \in \mathbb{J}_{\mathbb{T}}:=[0, \infty) \cap \mathbb{T}, t \neq \tau_{k}, t \geq t_{0},} \\
y\left(\tau_{k}^{+}\right)=g_{k}\left(y\left(\tau_{k}\right)\right), \quad y^{\Delta}\left(\tau_{k}^{+}\right)=h_{k}\left(y^{\Delta}\left(\tau_{k}\right)\right), \quad k \in \mathbb{N}, \\
y\left(t_{0}^{+}\right)=y_{0}, \quad y^{\Delta}\left(t_{0}^{+}\right)=y_{0}^{\Delta},
\end{array}\right.
$$

and improved the results of [13] and [14].

To the best of the author's knowledge, there is no such results for the impulsive delay dynamic equations on time scales. Hence, in this work an attempt is made to study the impulsive dynamic equations $(E)$ and from which we can find the corresponding results for impulsive differential/difference equation. In this direction, we refer the reader to some works ([2], [13]-[19]) and the references cited there in.

$A C^{i}=\left\{x: \mathbb{J}_{\mathbb{T}} \rightarrow \mathbb{R}\right.$ is $i$-times $\Delta$-differentiable, whose $i$ th delta derivative $x^{\Delta^{(i)}}$ is absolutely continuous $\}, P C=\left\{x: \mathbb{J}_{\mathbb{T}} \rightarrow \mathbb{R}\right.$ is rd-continuous at the points $\tau_{k}, k \in \mathbb{N}$ for which $x\left(\tau_{k}^{-}\right), x\left(\tau_{k}^{+}\right), x^{\Delta}\left(\tau_{k}^{-}\right)$and $x^{\Delta}\left(\tau_{k}^{+}\right)$exist, with $\left.x\left(\tau_{k}^{-}\right)=x\left(\tau_{k}\right), x^{\Delta}\left(\tau_{k}^{-}\right)=x^{\Delta}\left(\tau_{k}\right)\right\}$.

Definition 1.1. A solution of $x(t)$ of $(E)$ is said to be regular if it is defined on some half line $\left[\tau_{x}, \infty\right)_{\mathbb{T}} \subset\left[t_{0}, \infty\right)_{\mathbb{T}}$ and $\sup \left\{|x(t)|: t \geq t_{x}\right\}>0$. A regular solution $x(t)$ of $(E)$ is said to be eventually positive (eventually negative), if there exists $t_{1}>0$ such that $x(t)>0(x(t)<0)$ for $t \geq t_{1}$.

Definition 1.2. A function $x(t) \in P C \cap A C^{2}\left(\mathbb{J}_{\mathbb{T}} \backslash\left\{\tau_{1}, \tau_{2}, \ldots\right\}, \mathbb{R}\right)$ is called a solution of $(E)$ if:

(I) it satisfies $(E)$ a.e. on $\mathbb{J}_{\mathbb{T}} \backslash\left\{\tau_{k}\right\}, k \in \mathbb{N}$;

(II) for $t=\tau_{k}, k \in \mathbb{N}, x(t)$ satisfies $(E)$;

(III) for any $t \in\left[t_{0}-\delta, t_{0}\right], x(t)=\phi(t), x\left(t_{0}^{+}\right)=x_{0}, x^{\Delta}\left(t_{0}^{+}\right)=x_{0}^{\Delta}$. 
Definition 1.3. A nontrivial solution $x(t)$ of $(E)$ is said to be nonoscillatory, if there exists a point $t_{0} \geq 0$ such that $x(t)$ has a constant sign for $t \geq t_{0}$. Otherwise, the solution $x(t)$ is said to be oscillatory.

For completeness in the paper, we give the time scale concept and some fundamentals of time scale calculus in Section 4.

\section{BASIC LEMMAS}

We need the time scale version of the following well known results for our use in the sequel.

Lemma 2.1 ([1]). Let $y, f \in C_{r d}$ and $p \in \mathcal{R}$. Then $y^{\Delta}(t) \leq p(t) y(t)+f(t)$, implies that for all $t \in \mathbb{T}$

$$
y(t) \leq y\left(t_{0}\right) e_{p}\left(t, t_{0}\right)+\int_{t_{0}}^{t} e_{p}(t, \sigma(s)) f(s) \Delta s .
$$

Lemma 2.2 ([15]). Assume that

(i) $m \in P C \cap A C^{1}\left(\mathbb{J}_{\mathbb{T}} \backslash\left\{\tau_{k}\right\}, \mathbb{R}\right)$;

(ii) for $k \in \mathbb{N}$ and $t \geq t_{0}$, we have

$$
\begin{aligned}
& m^{\Delta}(t) \leq p(t) m(t)+v(t), \quad t \in \mathbb{J}_{\mathbb{T}}=[0, \infty) \cap \mathbb{T}, t \neq \tau_{k}, \\
& m\left(\tau_{k}^{+}\right) \leq d_{k} m\left(\tau_{k}\right)+e_{k} .
\end{aligned}
$$

Then the following inequality holds

$$
\begin{aligned}
m(t) \leq & m\left(t_{0}\right) \prod_{t_{0}<\tau_{k}<t} d_{k} e_{p}\left(t_{0}, t\right)+\int_{t_{0}}^{t} \prod_{s<\tau_{k}<t} d_{k} e_{p}(t, \sigma(s)) v(s) \Delta s \\
& +\sum_{t_{0}<\tau_{k}<t}\left(\prod_{\tau_{k}<\tau_{j}<t} d_{j} e_{p}\left(t, \tau_{k}\right)\right) e_{k}, t \geq t_{0} .
\end{aligned}
$$

Lemma 2.3. Suppose that $\left(A_{1}\right)-\left(A_{3}\right), a_{k}, b_{k}>0, k \in \mathbb{N}$ hold. Furthermore, assume that there exists $T \geq t_{0}$ such that $x(t)>0$ for $t \geq T$ and

$$
\left(A_{4}\right) \int_{T}^{\infty} \frac{1}{r^{\frac{1}{\gamma}}(s)} \prod_{T<\tau_{k}<s} \frac{b_{k}^{*}}{a_{k}} \Delta s=\infty .
$$

Then $x^{\Delta}\left(\tau_{k}^{+}\right) \geq 0$ and $x^{\Delta}(t) \geq 0$ for $t \in\left(\tau_{k}, \tau_{k+1}\right]_{\mathbb{T}}$ and $\tau_{k} \geq T$.

Proof. Let $x(t)$ be an eventually positive solution of $(E)$ for $t \geq t_{0}$. Without loss of generality we assume that $x(t)>0$ and $x(t-\delta)>0$ for $t \geq t_{1}>t_{0}+\delta$. From $(E)$, we get $\left[r(t)\left(x^{\Delta}(t)\right)^{\gamma}\right]^{\Delta}=-q(t) f(x(t-\delta)) \leq 0$. Therefore, $r(t)\left(x^{\Delta}(t)\right)^{\gamma}$ is monotonically decreasing on $\left[t_{2}, \infty\right)_{\mathbb{T}}, t_{2}>t_{1}+\delta$. Assume that $\tau_{k}>t_{2}$ for $k \in \mathbb{N}$. Consider the interval $\left(\tau_{k}, \tau_{k+1}\right]_{\mathbb{T}}, k \in \mathbb{N}$. We assert that $x^{\Delta}\left(\tau_{k}\right) \geq 0$. If not, there exists $\tau_{j} \geq t_{2}$ such that $x^{\Delta}\left(\tau_{j}\right)<0$ and hence $x^{\Delta}\left(\tau_{j}^{+}\right)=N_{k}\left(x^{\Delta}\left(\tau_{k}\right)\right) \leq b_{k}^{*} x^{\Delta}\left(\tau_{k}\right)<0$. Let $x^{\Delta}\left(\tau_{j}^{+}\right)=$ $-\alpha, \alpha>0$. Now for $t \in\left(\tau_{j}, \tau_{j+1}\right]_{\mathbb{T}}$, we have $r\left(\tau_{j+1}\right)\left(x^{\Delta}\left(\tau_{j+1}\right)\right)^{\gamma} \leq r\left(\tau_{j}\right)\left(x^{\Delta}\left(\tau_{j}^{+}\right)\right)^{\gamma}$, that is,

$$
x^{\Delta}\left(\tau_{j+1}\right) \leq\left(\frac{r\left(\tau_{j}\right)}{r\left(\tau_{j+1}\right)}\right)^{\frac{1}{\gamma}} x^{\Delta}\left(t_{j}^{+}\right)=-b_{j}^{*} \alpha\left(\frac{r\left(\tau_{j}\right)}{r\left(\tau_{j+1}\right)}\right)^{\frac{1}{\gamma}}<0 .
$$


If $t \in\left(\tau_{j+1}, \tau_{j+2}\right]_{\mathbb{T}}$, then

$$
\begin{aligned}
x^{\Delta}\left(\tau_{j+2}\right) & \leq\left(\frac{r\left(\tau_{j+1}\right)}{r\left(\tau_{j+2}\right)}\right)^{\frac{1}{\gamma}} x^{\Delta}\left(\tau_{j+1}^{+}\right)=\left(\frac{r\left(\tau_{j+1}\right)}{r\left(\tau_{j+2}\right)}\right)^{\frac{1}{\gamma}} N_{j+1}\left(x^{\Delta}\left(\tau_{j+1}\right)\right) \\
& \leq b_{j+1}^{*}\left(\frac{r\left(\tau_{j+1}\right)}{r\left(\tau_{j+2}\right)}\right)^{\frac{1}{\gamma}} x^{\Delta}\left(\tau_{j+1}\right),
\end{aligned}
$$

that is,

$$
x^{\Delta}\left(\tau_{j+2}\right) \leq-b_{j}^{*} b_{j+1}^{*} \alpha\left(\frac{r\left(\tau_{j}\right)}{r\left(\tau_{j+2}\right)}\right)^{\frac{1}{\gamma}}<0 .
$$

Hence, by the method of induction

$$
\begin{aligned}
x^{\Delta}\left(\tau_{j+n}\right) & \leq-b_{j}^{*} b_{j+1}^{*} b_{j+2}^{*} \cdots b_{j+n-1}^{*} \alpha\left(\frac{r\left(\tau_{j}\right)}{r\left(\tau_{j+n}\right)}\right)^{\frac{1}{\gamma}} \\
& =-\left(\frac{r\left(\tau_{j}\right)}{r\left(\tau_{j+n}\right)}\right)^{\frac{1}{\gamma}}\left(\prod_{i=1}^{n-1} b_{j+i}^{*}\right) \alpha<0,
\end{aligned}
$$

for $t \in\left(\tau_{j+n-1}, \tau_{j+n}\right]_{\mathbb{T}}$.

Now, we consider the following impulsive dynamic inequalities

$$
\left(E_{1}\right)\left\{\begin{array}{l}
{\left[r(t)\left(x^{\Delta}(t)\right)^{\gamma}\right]^{\Delta} \leq 0, \quad t>\tau_{j}, t \neq \tau_{k}, k=j+1, j+2, \ldots,} \\
x^{\Delta}\left(\tau_{k}^{+}\right) \leq b_{k}^{*} x^{\Delta}\left(\tau_{k}\right), \quad k=j+1, j+2, \ldots
\end{array}\right.
$$

Let $m(t)=r(t)\left(x^{\Delta}(t)\right)^{\gamma}$, then $\left(E_{1}\right)$ becomes

$$
\left\{\begin{array}{l}
m^{\Delta}(t) \leq 0, \quad t>\tau_{j}, t \neq \tau_{k}, k=j+1, j+2, \ldots, \\
m\left(\tau_{k}^{+}\right) \leq\left(b_{k}^{*}\right)^{\gamma} m\left(\tau_{k}\right), \quad k=j+1, j+2, \ldots,
\end{array}\right.
$$

and, by Lemma 2.2, it follows that

$$
m(t) \leq m\left(\tau_{j}^{+}\right) \prod_{\tau_{j}<\tau_{k}<t}\left(b_{k}^{*}\right)^{\gamma},
$$

that is,

$$
x^{\Delta}(t) \leq\left(\frac{r\left(\tau_{j}\right)}{r(t)}\right)^{\frac{1}{\gamma}} x^{\Delta}\left(\tau_{j}^{+}\right) \prod_{\tau_{j}<\tau_{k}<t} b_{k}^{*}=-\alpha\left(\frac{r\left(\tau_{j}\right)}{r(t)}\right)^{\frac{1}{\gamma}} \prod_{\tau_{j}<\tau_{k}<t} b_{k}^{*} .
$$

For $k=j+1, j+2, \ldots$, we also have $x\left(\tau_{k}^{+}\right) \leq a_{k} x\left(\tau_{k}\right)$. By $(2.1)$ and since $x\left(\tau_{k}^{+}\right) \leq$ $a_{k} x\left(\tau_{k}\right), k=j+1, j+2, \ldots$, it follows from Lemma 2.2 that

$$
\begin{aligned}
x(t) & \leq x\left(\tau_{j}^{+}\right) \prod_{\tau_{j}<\tau_{k}<t} a_{k}-\int_{\tau_{j}}^{t} \prod_{s<\tau_{k}<t} a_{k}\left[\alpha\left(\frac{r\left(\tau_{j}\right)}{r(t)}\right)^{\frac{1}{\gamma}} \prod_{\tau_{j}<\tau_{k}<s} b_{k}^{*}\right] \Delta s \\
& \leq \prod_{\tau_{j}<\tau_{k}<t} a_{k}\left[x\left(\tau_{j}^{+}\right)-\alpha\left(r\left(\tau_{j}\right)\right)^{\frac{1}{\gamma}} \int_{\tau_{j}}^{t}\left(\frac{1}{r(s)}\right)^{\frac{1}{\gamma}} \prod_{\tau_{j}<\tau_{k}<s} \frac{b_{k}^{*}}{a_{k}} \Delta s\right]
\end{aligned}
$$


$\rightarrow-\infty$ as $t \rightarrow \infty$

Due to $\left(A_{4}\right)$, a contradiction to the fact that $x(t)>0$ eventually. Hence, our assertation holds, that is, $x^{\Delta}\left(\tau_{k}\right) \geq 0$ for $\tau_{k} \geq T$ and hence $x^{\Delta}(t)>x^{\Delta}\left(\tau_{k}^{+}\right)$. Since $\left[r(t)\left(x^{\Delta}(t)\right)^{\gamma}\right]^{\Delta} \leq 0$ for any $t \in\left(\tau_{k}, \tau_{k+1}\right]_{\mathbb{T}}, \tau_{k} \geq T$, then

$$
x^{\Delta}(t) \geq\left(\frac{r\left(\tau_{k+1}\right)}{r(t)}\right)^{\frac{1}{\gamma}} x^{\Delta}\left(\tau_{k+1}\right) \geq 0, \quad t \in\left(\tau_{k}, \tau_{k+1}\right]_{\mathbb{T}} .
$$

Therefore, $x^{\Delta}\left(\tau_{k}^{+}\right)>0$ and $x^{\Delta}(t)>0$ for $\left.t \in\left(\tau_{k}, \tau_{k+i}\right)\right]_{\mathbb{T}}, t \geq t_{2}$, and the lemma is proved.

Remark 2.1. If $x(t)$ is an eventually negative solution of $(E)$. Then, using $\left(A_{1}\right)-\left(A_{3}\right)$, it is easy to prove that $x^{\Delta}\left(\tau_{k}^{+}\right) \leq 0$ and $x^{\Delta}(t) \leq 0$, for $t \in\left(\tau_{k}, \tau_{k+1}\right]_{\mathbb{T}}$ and $\tau_{k} \geq T \geq t_{0}$.

\section{Sufficient Conditions for Oscillation}

Theorem 3.1. Let all conditions of Lemma 2.3 hold. Furthermore, assume that

$$
\left(A_{5}\right) \int_{t_{0}}^{\infty} \prod_{t_{0}<\tau_{k}<s} \frac{1}{b_{k}^{\gamma}} q(s) \Delta s=\infty .
$$

Then every solution of $(E)$ oscillates.

Proof. Suppose on the contrary that $x(t)$ is a nonoscillatory solution of $(E)$. Without loss of generality, assume that $x(t)>0, x(\sigma(t)-\delta)>0$ for $t \geq t_{1}$. Hence, by Lemma 2.3 , there exists $t_{2}>t_{1}$ such that $x^{\Delta}(t)>0$ for $t \in\left(\tau_{k}, \tau_{k+1}\right]_{\mathbb{T}}, k \in \mathbb{N}$ and $\tau_{k} \geq t_{2}$. Indeed, $x^{\Delta}(t-\delta)>0$ for $t \geq t_{3} \geq t_{2}+\delta$. Let

$$
w(t)=\frac{r(t)\left(x^{\Delta}(t)\right)^{\gamma}}{x(t-\delta)} .
$$

Then $w\left(\tau_{k}^{+}\right) \geq 0$ and $w(t) \geq 0$ for $\tau_{k} \geq t_{3}$. From (3.1), for $t \neq \tau_{k}$ we have

$$
\begin{aligned}
w^{\Delta}(t) & =\frac{\left[r(t)\left(x^{\Delta}(t)\right)^{\gamma}\right]^{\Delta} x(t-\delta)-r(\sigma(t))\left(x^{\Delta}(\sigma(t))\right)^{\gamma} x^{\Delta}(t-\delta)}{x(t-\delta) x(\sigma(t)-\delta)} \\
& \leq \frac{\left[r(t)\left(x^{\Delta}(t)\right)^{\gamma}\right]^{\Delta}}{x(\sigma(t)-\delta)}-\frac{r(\sigma(t))\left(x^{\Delta}(\sigma(t))\right)^{\gamma} x^{\Delta}(t-\delta)}{x(t-\delta) x(\sigma(t)-\delta)} \\
& \leq-q(t),
\end{aligned}
$$

that is,

$$
w^{\Delta}(t) \leq-q(t), \quad t \neq \tau_{k}
$$

We note that

$$
w\left(\tau_{k}^{+}\right)=\frac{r\left(\tau_{k}^{+}\right)\left(x^{\Delta}\left(\tau_{k}^{+}\right)\right)^{\gamma}}{x\left(\tau_{k}^{+}-\delta\right)} \leq \frac{b_{k}^{\gamma} r\left(\tau_{k}\right)\left(x^{\Delta}\left(\tau_{k}\right)\right)^{\gamma}}{x\left(\tau_{k}-\delta\right)}=b_{k}^{\gamma} w\left(\tau_{k}\right) .
$$

Now, we have the following impulsive dynamics inequalities

$$
\begin{gathered}
w^{\Delta}(t) \leq-q(t), \quad t \neq \tau_{k} \\
w\left(\tau_{k}^{+}\right) \leq b_{k}^{\gamma} w\left(\tau_{k}\right), \quad k \in \mathbb{N},
\end{gathered}
$$


and, by Lemma 2.2, it follows that

$$
\begin{aligned}
w(t) & \leq w\left(t_{3}\right) \prod_{t_{3}<\tau_{k}<t} b_{k}^{\gamma}-\int_{t_{3}}^{t} \prod_{s<\tau_{k}<t} b_{k}^{\gamma} q(s) \Delta s \\
& \leq \prod_{t_{3}<\tau_{k}<t} b_{k}^{\gamma}\left[w\left(t_{3}\right)-\int_{t_{3}}^{t} \prod_{t_{3}<\tau_{k}<s} \frac{1}{b_{k}^{\gamma}} q(s) \Delta s\right] \\
& \rightarrow-\infty \text { as } t \rightarrow \infty .
\end{aligned}
$$

Due to $\left(A_{5}\right)$, a contradiction to the fact that $w(t)>0$ for $t \in\left(\tau_{k}, \tau_{k+1}\right]_{\mathbb{T}}, k \in \mathbb{N}$. This completes the proof of the theorem.

Theorem 3.2. Let all conditions of Lemma 2.3 hold. Furthermore, assume that $\tau_{k+1}-\tau_{k}=\delta$ and

$\left(A_{6}\right) \int_{t_{0}}^{\infty} \prod_{t_{0}<\tau_{k}<s} \frac{1}{d_{k}} q(s) \Delta s=\infty$,

where

$$
d_{k}= \begin{cases}b_{1}^{\gamma}, & \text { if } k=1, \\ d \frac{b_{k}^{\gamma}}{a_{k-1}^{*}}, & \text { if } k=2,3, \ldots,\end{cases}
$$

hold. Then every solution of $(E)$ oscillates.

Proof. Proceed as in the proof Theorem 3.1 to obtain that $x^{\Delta}(t)>0$ and $x^{\Delta}\left(\tau_{k}^{+}\right)>0$ for $t \in\left(\tau_{k}, \tau_{k+1}\right]_{\mathbb{T}}, k \in \mathbb{N}, t \geq t_{2}$. Indeed, $x^{\Delta}(t-\delta)>0$ for $t \geq t_{3} \geq t_{2}+\delta$. Define $w(t)$ as in (3.1), we get (3.2) holds for $\tau_{k} \geq t_{3}$ and $t \neq \tau_{k}$. Now, if $k=1$ we have

$$
w\left(\tau_{1}^{+}\right)=\frac{r\left(\tau_{1}^{+}\right)\left(x^{\Delta}\left(\tau_{1}^{+}\right)\right)^{\gamma}}{x\left(\tau_{1}^{+}-\delta\right)} \leq \frac{b_{1}^{\gamma} r\left(\tau_{1}\right)\left(x^{\Delta}\left(\tau_{1}\right)\right)^{\gamma}}{x\left(\tau_{1}-\delta\right)}=d_{1} w\left(\tau_{1}\right) .
$$

If $k=2,3, \ldots$, then

$$
\begin{aligned}
w\left(\tau_{k}^{+}\right) & =\frac{r\left(\tau_{k}^{+}\right)\left(x^{\Delta}\left(\tau_{k}^{+}\right)\right)^{\gamma}}{x\left(\tau_{k}^{+}-\delta\right)} \leq \frac{b_{k}^{\gamma} r\left(\tau_{k}\right)\left(x^{\Delta}\left(\tau_{k}\right)\right)^{\gamma}}{x\left(\tau_{k-1}^{+}-\delta\right)} \leq \frac{b_{k}^{\gamma} r\left(\tau_{k}\right)\left(x^{\Delta}\left(\tau_{k}\right)\right)^{\gamma}}{a_{k-1}^{*} x\left(\tau_{k-1}-\delta\right)} \\
& \leq \frac{b_{k}^{\gamma} r\left(\tau_{k}\right)\left(x^{\Delta}\left(\tau_{k}\right)\right)^{\gamma}}{a_{k-1}^{*} x\left(\tau_{k}-\delta\right)}=d_{k} w\left(\tau_{k}\right) .
\end{aligned}
$$

Consider the following impulsive dynamic inequality

$$
\left\{\begin{array}{l}
w^{\Delta}(t) \leq-q(t), \quad t \neq \tau_{k}, t \geq t_{3} \\
w\left(\tau_{k}^{+}\right) \leq d_{k} w\left(\tau_{k}\right), \quad k \in \mathbb{N} .
\end{array}\right.
$$

Therefore, by Lemma 2.2, we get

$$
w(t) \leq w\left(t_{3}\right) \prod_{t_{3}<\tau_{k}<t} d_{k}-\int_{t_{3}}^{t} \prod_{u<\tau_{k}<t} d_{k} q(u) \Delta u .
$$

Then proceeding as in the proof of Theorem 3.1 and using $\left(A_{6}\right)$, we get a contradiction to the fact that $w(t)>0$ for $t \in\left(\tau_{k}, \tau_{k+1}\right]_{\mathbb{T}}, k \in \mathbb{N}$. This completes the proof of the theorem. 
Corollary 3.1. Let all conditions of Lemma 2.3 hold. Assume that there exists a positive integer $k_{0}$ such that $a_{k}^{*} \geq 1, b_{k} \leq 1$ for $k \geq k_{0}$. Furthermore, assume that

$\left(A_{7}\right) \int_{t_{0}}^{\infty} q(s) \Delta s=\infty$

holds, then every solution of $(E)$ oscillates.

Proof. Without loss of generality, we assume that $k_{0}=1$. Since $b_{k} \leq 1$, then $\frac{1}{b_{k}^{\gamma}} \geq 1$. Therefore,

$$
\int_{t_{0}}^{t} \prod_{t_{0} \leq \tau_{k}<s} \frac{1}{b_{k}^{\gamma}} q(s) \Delta s \geq \int_{t_{0}}^{t} q(s) \Delta s .
$$

Letting $t \rightarrow \infty$ and in view of Theorem 3.1, We get every solution of $(E)$ is oscillatory. This completes the proof.

Corollary 3.2. Let all conditions of Lemma 2.3 hold. Assume that there exists a positive integer $k_{0}$ and a positive constant $\alpha$ such that $a_{k}^{*} \geq 1$ and $\frac{1}{b_{k}} \geq\left(\frac{\tau_{k+1}}{\tau_{k}}\right)^{\alpha}$ for $k \geq k_{0}$. Furthermore, assume that

$$
\left(A_{8}\right) \int_{t_{0}}^{\infty} s^{\alpha} q(s) \Delta s=\infty
$$

holds, then every solution of $(E)$ oscillates.

Proof. Without loss of generality, we assume that $k_{0}=1$. Now

$$
\begin{aligned}
\int_{t_{0}}^{t} \prod_{t_{0}<\tau_{k}<s} \frac{1}{b_{k}^{\gamma}} q(s) \Delta s & =\sum_{i=1}^{n} \prod_{t_{0}<\tau_{k}<\tau_{i+1}} \frac{1}{b_{k}^{\gamma}} \int_{\tau_{i}}^{\tau_{i+1}} q(s) \Delta s \\
& \geq \frac{1}{\tau_{1}^{\alpha}} \sum_{i=1}^{n} \tau_{i+1}^{\alpha} \int_{\tau_{i}}^{\tau_{i+1}} q(s) \Delta s \\
& \geq \frac{1}{\tau_{1}^{\alpha}} \sum_{i=1}^{n} \int_{\tau_{i}}^{\tau_{i+1}} s^{\alpha} q(s) \Delta s \\
& =\frac{1}{\tau_{1}^{\alpha}} \int_{\tau_{1}}^{\tau_{n+1}} s^{\alpha} q(s) \Delta s .
\end{aligned}
$$

Letting $t \rightarrow \infty$ and in view of Theorem 3.1, we get every solution of $(E)$ is oscillatory. This completes the proof.

Corollary 3.3. Let all conditions of Lemma 2.3 hold. Assume that there exists a positive integer $k_{0}$ and a positive constant $\alpha$ such that $a_{k}^{*} \geq 1$ and $\frac{1}{d_{k}} \geq\left(\frac{\tau_{k+1}}{\tau_{k}}\right)^{\alpha}$ for $k \geq k_{0}$. If $\left(A_{8}\right)$ hold, then every solution of $(E)$ oscillates.

Proof. The proof of the corollary can be be follows from Corollary 3.2 and Theorem 3.2. Hence, details are omitted.

Next, we present some new oscillation criteria for $(E)$, by using an integral averaging condition of Kamenev type.

Theorem 3.3. Let all the conditions of Lemma 2.3 and $b_{k} \geq 1$ hold. Furthermore, assume that 
$\left(A_{9}\right) \lim \sup _{k \rightarrow \infty} \frac{1}{t^{m}} \int_{t_{0}}^{\tau_{k+1}}(t-s)^{m} q(s) \Delta s=\infty$, then every solution of $(E)$ oscillates.

Proof. Proceeding as in the proof of Theorem 3.1, we get

$$
w^{\Delta}(t) \leq-q(t), \quad \text { for } t \neq \tau_{k} .
$$

Multiplying $(t-s)^{m}$ to both side of the preceding inequality and integrating from $\tau_{k}$ to $\tau_{k+1}$, we get

$$
\int_{\tau_{k}}^{\tau_{k+1}}(t-s)^{m} w^{\Delta}(s) d s \leq-\int_{\tau_{k}}^{\tau_{k+1}}(t-s)^{m} q(s) \Delta s .
$$

Indeed,

$$
\begin{aligned}
& \int_{\tau_{k}}^{\tau_{k+1}}(t-s)^{m} w^{\Delta}(s) \Delta s \\
= & \left.(t-s)^{m} u(s)\right|_{\tau_{k}} ^{\tau_{k+1}}-\int_{\tau_{k}}^{\tau_{k+1}}\left((t-s)^{m}\right)^{\Delta_{s}} w(s) \Delta s \\
= & \int_{\tau_{k}}^{\tau_{k+1}} m(t-s)^{m-1} w(s) \Delta s+\left(t-\tau_{k+1}\right)^{m} w\left(\tau_{k+1}\right)-\left(t-\tau_{k}\right)^{m} w\left(\tau_{k}^{+}\right),
\end{aligned}
$$

because $\left((t-s)^{m}\right)^{\Delta_{s}}=-m(t-s)^{m-1}$. As a result,

$$
\int_{\tau_{k}}^{\tau_{k+1}}(t-s)^{m} w^{\Delta}(s) \Delta s \geq-\left(t-\tau_{k}\right)^{m} w\left(\tau_{k}^{+}\right) .
$$

Therefore,

$$
\begin{aligned}
\int_{\tau_{k}}^{\tau_{k+1}}(t-s)^{m} q(s) \Delta s & \leq-\int_{\tau_{k}}^{\tau_{k+1}}(t-s)^{m} w^{\Delta}(s) \Delta s \\
& \leq\left(t-\tau_{k}\right)^{m} w\left(\tau_{k}^{+}\right) \\
& \leq b_{k}\left(t-\tau_{k}\right)^{m} w\left(\tau_{k}\right),
\end{aligned}
$$

that is,

$$
\frac{1}{t^{m}} \int_{\tau_{k}}^{\tau_{k+1}}(t-s)^{m} q(s) \Delta s \leq b_{k}\left(\frac{t-\tau_{k}}{t}\right)^{m} w\left(\tau_{k}\right),
$$

and hence,

$$
\limsup _{k \rightarrow \infty} \frac{1}{t^{m}} \int_{\tau_{k}}^{\tau_{k+1}}(t-s)^{m} q(s) \Delta s<\infty
$$

a contradiction to $\left(A_{9}\right)$. This completes the proof of the theorem.

\section{Appendix: Time Scale Preliminaries}

We will briefly recall some basic definitions and facts from the time scale calculus that we will use in the sequel. For more details see $[2,3,19]$. On any time scale $\mathcal{T}$, we define the forward and backward jump operators by

$$
\sigma(t)=\inf \{s \in \mathbb{T}: s>t\}, \quad \rho(t)=\sup \{s \in \mathbb{T}: V s<t\},
$$


where $\inf \phi=\sup \mathbb{T}, \sup \phi=\inf \mathbb{T}$, and $\phi$ denotes the empty set. A nonmaximal element $t \in \mathbb{T}$ is called right-dense if $\sigma(t)=t$ and right-scattered if $\sigma(t)>t$. A nonminimal element $t \in \mathbb{T}$ is said to be left-dense if $\rho(t)=t$ and left-scattered if $\rho(t)>t$. The graininess $\mu$ of the time scale $\mathbb{T}$ is defined by $\mu(t)=\sigma(t)-t$.

A mapping $f: \mathbb{T} \rightarrow \mathbb{X}$ is said to be differentiable at $t \in \mathbb{T}$, if there exists $f^{\Delta}(t) \in \mathbb{X}$ such that for any $\epsilon>0$, there exists a neighborhood $U$ of $t$ satisfying

$$
\left|[f(\sigma(t))-f(s)]-f^{\Delta}(t)[\sigma(t)-s]\right| \leq \epsilon|\sigma(t)-s|,
$$

for all $s \in U$. We say that $f$ is delta differentiable (or in short: differentiable) on $\mathbb{T}$ provided $f^{\Delta}(t)$ exists for all $t \in \mathbb{T}$.

A function $f: \mathbb{T} \rightarrow \mathbb{R}$ is called rd-continuous provided it is continuous at right-dense points in $\mathbb{T}$ and its left-sided limits exist (finite) at left-dense points in $\mathbb{T}$. The set of rd-continuous functions $f: \mathbb{T} \rightarrow \mathbb{R}$ will be denoted by $C_{r d}(\mathbb{T}, \mathbb{R})$.

The derivative and forward jump operator $\sigma$ are related by the formula

$$
f(\sigma(t))=f(t)+\mu(t) f^{\Delta}(t) .
$$

Let $f$ be a differentiable function on $[a, b]_{\mathbb{T}}$. Then $f$ is increasing, decreasing, nondecreasing and nonincreasing on $[a, b]_{\mathbb{T}}$ if $f^{\Delta}>t, f^{\Delta}<t, f^{\Delta} \geq t, f^{\Delta} \leq t$ for all $t \in[a, b)_{\mathbb{T}}$, respectively. We will make use of the following product $f g$ and quotient $\frac{f}{g}$ rules for the derivative of two differentiable functions $f$ and $g$

$$
\begin{aligned}
& (f g)^{\Delta}=f^{\Delta} g+f^{\sigma} g^{\Delta}=f g^{\Delta}+f^{\Delta} g^{\sigma}, \\
& \left(\frac{f}{g}\right)^{\Delta}=\frac{f^{\Delta} g-f g^{\Delta}}{g g^{\sigma}},
\end{aligned}
$$

where $f^{\sigma}=f_{o \sigma}, g g^{\sigma} \neq 0$. The integration by parts formula reads

$$
\int_{a}^{b} f^{\Delta}(t) g(t)=\left.f(t) g(t)\right|_{a} ^{b}-\int_{a}^{b} f^{\sigma}(t) g^{\Delta}(t) \Delta t
$$

Chain Rule. Assume $g: \mathbb{T} \rightarrow \mathbb{R}$ is $\Delta$ - differentiable on $\mathbb{T}$ and $f: \mathbb{R} \rightarrow \mathbb{R}$ is continuously differentiable. Then $f \circ g: \mathbb{T} \rightarrow \mathbb{R}$ is $\Delta$-differentiable and satisfies

$$
(f \circ g)^{\Delta}(t)=\left\{\int_{0}^{1} f^{\prime}\left(g(t)+h \mu(t) g^{\Delta}(t)\right) d h\right\} g^{\Delta}(t) .
$$

Regressive. A function $p: \mathbb{T} \rightarrow \mathbb{R}$ is said to be regressive if for all $t \in \mathbb{T}, 1+\mu(t) p(t) \neq$ 0 .

The set of all function $p: \mathbb{T} \rightarrow \mathbb{R}$, which are regressive and rd-continuous will be denoted by $\mathcal{R}$. We define the set $\mathcal{R}^{+}$of all positively regressive elements of $\mathcal{R}$ by

$$
\mathcal{R}^{+}=\{p \in \mathcal{R}: 1+\mu(t) p(t)>0 \text { for all } t \in \mathbb{T}\} .
$$

Exponential Function. If $p \in \mathcal{R}$, then general exponential function $e_{p}$ on $\mathbb{T}$ is defined as

$$
e_{p}(t, s)=\exp \left(\int_{s}^{t} \frac{1}{\mu(z)} \log (1+\mu(z) p(z)) \Delta z\right),
$$

with $\mu(z) \neq 0$ and $s, t \in \mathbb{T}$. 


\section{Examples}

Example 5.1. Consider the impulsive dynamic equation

$$
\left\{\begin{array}{l}
x^{\Delta \Delta}(t)+\frac{1}{t} x\left(t-\frac{1}{2}\right)=0, \quad t>\frac{1}{2}, t \neq \tau_{k}, \\
x\left(\tau_{k}^{+}\right)=\frac{k+1}{k} x\left(\tau_{k}\right), \quad x^{\Delta}\left(\tau_{k}^{+}\right)=x^{\Delta}\left(\tau_{k}\right), \quad k \in \mathbb{N},
\end{array}\right.
$$

where $\gamma=1, r(t)=1, \delta=\frac{1}{2}, q(t)=\frac{1}{t} \geq 0, a_{k}^{*}=a_{k}=\frac{k+1}{k}, b_{k}^{*}=b_{k}=1, \tau_{k}=3 k$, $\tau_{k+1}-\tau_{k}=3>2, k \in \mathbb{N}$. Then, from $\left(A_{4}\right)$

$$
\begin{aligned}
& \int_{T}^{\infty} \prod_{T<\tau_{k}<s} \frac{b_{k}^{*}}{a_{k}} \Delta s \\
= & \int_{2}^{\infty} \prod_{2<\tau_{k}<s} \frac{k}{k+1} d s \\
= & \int_{2}^{\tau_{1}} \prod_{2<\tau_{k}<s} \frac{k}{k+1} \Delta s+\int_{\tau_{1}^{+}}^{\tau_{2}} \prod_{2<\tau_{k}<s} \frac{k}{k+1} \Delta s+\int_{\tau_{2}^{+}}^{\tau_{3}} \prod_{2<\tau_{k}<s} \frac{k}{k+1} \Delta s+\cdots \\
= & \frac{1}{2}\left(\tau_{1}-2\right)+\frac{1}{2} \times \frac{2}{3}\left(\tau_{2}-\tau_{1}\right)+\frac{1}{2} \times \frac{2}{3} \times \frac{3}{4}\left(\tau_{3}-\tau_{2}\right)+\cdots \\
= & \frac{1}{2} \times 2+\frac{1}{3} \times 3+\frac{1}{4} \times 3+\frac{1}{5} \times 3+\cdots \\
\geq & \frac{1}{2}+\frac{1}{3}+\frac{1}{4}+\frac{1}{5}+\cdots=\sum_{i=2}^{\infty} \frac{1}{i}=\infty,
\end{aligned}
$$

and from $\left(A_{5}\right)$

$$
\int_{2}^{\infty} \prod_{\frac{1}{2}<\tau_{k}<s} \frac{1}{b_{k}^{\gamma}} \frac{1}{s} \Delta s=\int_{2}^{\infty} \frac{1}{s} \Delta s \rightarrow \infty .
$$

Therefore, all conditions of Theorem 3.1 are satisfied and hence (5.1) has an oscillatory solution.

Example 5.2. Consider the impulsive dynamic equation

$$
\left\{\begin{array}{l}
x^{\Delta \Delta}(t)+\frac{1}{t^{3}} x(t-1)=0, \quad t>1, t \neq \tau_{k}, \\
x\left(\tau_{k}^{+}\right)=\frac{k-1}{k} x\left(\tau_{k}\right), \quad k \in \mathbb{N}, k>k_{0}, \\
x^{\Delta}\left(\tau_{k}^{+}\right)=\frac{1}{k} x^{\Delta}\left(\tau_{k}\right), \quad k \in \mathbb{N}, k>k_{0},
\end{array}\right.
$$

where $\gamma=1, \delta=1, r(t)=1, q(t)=\frac{1}{t^{3}} \geq 0, a_{k}^{*}=a_{k}=\frac{k-1}{k}, b_{k}^{*}=b_{k}=\frac{1}{k}, \tau_{k}=3 k$, $\tau_{k+1}-\tau_{k}=3>1, k \in \mathbb{N}, k>k_{0}=1$. Clearly, from $\left(A_{4}\right)$ we have

$$
\int_{T}^{\infty} \prod_{T<\tau_{k}<s} \frac{b_{k}^{*}}{a_{k}} \Delta s
$$




$$
\begin{aligned}
& =\int_{1}^{\infty} \prod_{1<\tau_{k}<s} \frac{1}{k-1} \Delta s \\
& =\int_{1}^{\tau_{2}} \prod_{1<\tau_{k}<s} \frac{1}{k-1} \Delta s+\int_{\tau_{2}^{+}}^{\tau_{3}} \prod_{1<\tau_{k}<s} \frac{1}{k-1} \Delta s+\int_{\tau_{3}^{+}}^{\tau_{4}} \prod_{1<\tau_{k}<s} \frac{1}{k-1} \Delta s+\cdots \\
& =\left(\tau_{2}-1\right)+\frac{1}{2} \times\left(\tau_{3}-\tau_{2}\right)+\frac{1}{2} \times \frac{1}{3} \times\left(\tau_{4}-\tau_{3}\right)+\cdots \\
& =2+\frac{1}{2} \times 2^{2}+\frac{1}{2} \times \frac{1}{3} \times 2^{3}+\frac{1}{2} \times \frac{1}{3} \times \frac{1}{4} \times 2^{4}+\cdots \\
& \geq 1+\frac{1}{2}+\frac{1}{3}+\frac{1}{4}+\cdots=1+\sum_{i=2}^{\infty} \frac{1}{i}=\infty .
\end{aligned}
$$

Let $\alpha=1$. Then

$$
\frac{1}{b_{k}}=k \geq\left(\frac{\tau_{k+1}}{\tau_{k}}\right)^{\alpha}=\frac{k+1}{k} .
$$

Also, from $\left(A_{8}\right)$ we have

$$
\int_{1}^{\infty} s^{\alpha} q(s) \Delta s=\int_{1}^{\infty} s^{3} \frac{1}{s^{3}} \Delta s=\int_{1}^{\infty} \Delta s=\infty .
$$

All conditions of Corollary 3.2 are satisfied for (5.2) and hence, (5.2) has an oscillatory solution.

Acknowledgements. This work is supported by Rajiv Gandhi National fellowship(UGC), New Delhi, India, through the Letter No. F1-17.1/2017-18 RGNF-201718-SC-ORI-35849, dated 11th July, 2017.

We are very thankful to the anonymous reviewers and editor for their constructive comments and suggestions which help us to improve the manuscript

\section{REFERENCES}

[1] R. P. Agarwal, M. Bohner and A. Peterson, Inequality on time scales: a survey, Math. Inequal. Appl. 4 (2001), 555-557.

[2] H. A. Agwa, A. M. M. Khodier and H. M. Atteya, Oscillation of second order nonlinear impulsive dynamic equations on time scales, Journal of Analysis \& Number Theory 5 (2017), 147-154.

[3] A. Belarbi, M. Benchohra and A. Ouahab, Extremal solutions for impulsive dynamic equations on time scales, Comm. Appl. Nonlinear Anal. 12 (2005), 85-95.

[4] M. Benchohra, J. Henderson, S. K. Ntouyas and A. Ouahab, On first order impulsive dynamic equations on time scales, J. Difference Equ. Appl. 10 (2004), 541-548.

[5] M. Benchohra, S. K. Ntouyas and A. Ouahab, Extremal solutions of second order impulsive dynamic equations on time scales, J. Math. Anal. Appl. 324 (2006), 425-434.

[6] M. Bohner and A. Peterson, Dynamic Equations on Time Scales: An Introduction with Applications, Birkhauser, Boston, 2001.

[7] M. Bohner and A. Peterson, Advances in Dynamic Equations on Time Scales, Birkhauser, Boston, 2003.

[8] M. Bohner and C. Tisdell, Oscillation and nonoscillation of forced second order dynamic equations, Pacific J. Math. 230 (2007), 59-71. 
[9] Y. K. Chang and W. T. Li, Existence results for impulsive dynamic equations on time scales with nonlocal initial conditions, Math. Comput. Model. Dyn. Syst. 43 (2006), 337-384.

[10] Z. He and W. Ge, Oscillation of impulsive delay differential equations, Indian J. Pure Appl. Math. 31 (2000), 1089-1101.

[11] Z. He and W. Ge, Oscillation in second order linear delay differential equations with nonlinear impulses, Math. Slovaca 52 (2002), 331-341.

[12] S. Hilger, Analysis on measure chains-a unified approach to continuous and discrete calculus, Results Math. 18 (1990), 18-56.

[13] M. Huang and W. Feng, Oscillation of second order nonlinear impulsive dynamic equations on time scales, Electron. J. Differential Equations 72 (2007), 1-13.

[14] M. Huang and W. Feng, Oscillation criteria for impulsive dynamic equations on time scales, Differ. Equ. 169 (2007), 1-9.

[15] M. Huang, Oscillation criteria for second order nonlinear dynamic equations with impulses, Comput. Math. Appl. 59 (2010), 31-41.

[16] E. R. Kaufmann, N. Kosmatov and Y. N. Raffoul, Impulsive dynamic equations on a time scale, Electron. J. Differential Equations 69 (2008), 1-9.

[17] Q. Li and F. Guo, Oscillation of solutions to impulsive dynamic equations on time scales, Electron. J. Differential Equations 122 (2009), 1-7.

[18] Q. Li and L. Zhou, Oscillation criteria for second order impulsive dynamic equations on time scales, Appl. Math. E-Notes 11 (2011), 33-40.

[19] M. Peng, Oscillation Criteria for second order impulsive delay difference equations, Appl. Math. Comput. 146 (2003), 227-235.

[20] S. Sun, Z. Han and C. Zhang, Oscillation of second order delay dynamic equations on time scales, J. Appl. Math. Comput. 30 (2009), 459-468.

[21] Q. Zhang, L. Gao and L. Wang, Oscillation of second order nonlinear delay dynamic equations on time scales, Comput. Math. Appl. 61 (2011), 2342-2348.

\footnotetext{
${ }^{1}$ Department Of Mathematics,

SAMBALPUR UNIVERSITY

SAMBALPUR-768019, INDIA

Email address: c.gokulananda@gmail.com
} 